\title{
THE FUNDAMENTAL GROUP OF THE SPACE OF MAPS FROM A SURFACE INTO THE PROJECTIVE PLANE
}

\author{
DACIBERG L. GONÇALVES and MAURO SPREAFICO
}

\begin{abstract}
In this work we compute the fundamental group of each connected component of the function space of maps from a closed surface into the projective space
\end{abstract}

\section{Introduction}

The homotopy of the function spaces $S_{2}^{S_{1}}$ of continuous maps from a surface $S_{1}$ to another surface $S_{2}$ has been studied at least in the past 50 years. The problem can be divided into two cases. The first case is when the surface $S_{2}$ is a $K(\pi, 1)$, i.e. different from $S^{2}$ and $\mathrm{R} P^{2}$. In this situation the problem has been solved completely. See [3], [5], [6], and [7] (see also related works on the homotopy type of the components of function spaces [8], [10], [12]). The other case is when the target is either the sphere $S^{2}$ or the projective plane. For $S_{2}=S^{2}$, some relevant results were obtained. In [4] the fundamental group of each connected component of $\left(S^{2}\right)^{S_{1}}$ was determined up to some extension. In [9] these groups were completely determined. Finally in [7] a further homotopy information was given at least for the case of the components of $\left(S^{2}\right)^{S^{2}}$. Namely, for each degree $k$, the component which corresponds to this degree has a unique $k$-fold covering space, and this covering space has the homotopy type of the space of orientation preserving self-homotopy equivalences of $S^{2}$. Among several open questions about these function spaces, it seems to us that the question still remains open to find the fundamental group of the function space when the target is $\mathrm{R} P^{2}$. For the case when the target is $\mathrm{R} P^{2}$ there are few results. From [9] one can read the fundamental group of $\left(\mathrm{R} P^{2}\right)^{S}$ at least in the case of the connected component of the constant map. When also $S=\mathrm{R} P^{2}$, for the component of the identity map, namely for the space of self homotopy equivalences of the projective space $m_{*}\left(\mathrm{R} P^{2}, \mathrm{R} P^{2} ; \mathrm{id}\right)$, we can find in [13] a splitting similar to the one obtained in [6] for the sphere. This information can 
be used in order to compute the fundamental group of this component, provided we can compute the fundamental group of the space of based self homotopy equivalences (see details at the end of Section 3). The purpose of this note is to compute the fundamental group in the remaining cases, i.e. the fundamental group of the connected components which contain a map $f: S \rightarrow \mathrm{R} P^{2}$ that is not homotopic to the constant map, for a generic surface $S$.

\section{Background and notation}

In this section we introduce some notation and we recall some general facts and some results from works of P. Olum [11] and of Larmore and Thomas [9] that will be used in the following sections. For the definition of degree of a map see [2]. To start, we briefly describe the set of the components of the function space $\left(\mathrm{R} P^{2}\right)^{S}$. Our description is based on [11]. First, we fix some notation.

- $S$ will be a compact connected surface without boundary.

- We will use multiplicative notation for the fundamental group and additive notation for cohomology groups.

- We have an isomorphism between the groups $\operatorname{Hom}\left(\pi_{1}(S), \pi_{1}\left(\mathrm{R} P^{2}\right)\right)$ and $H^{1}(S ; \mathbf{Z} / 2)$. We will use $\theta$ to denote homomorphisms and $x$ to denote classes.

- An element of $\operatorname{Hom}\left(\pi_{1}(S), \pi_{1}\left(\mathrm{R} P^{2}\right)\right)$ is said to be orientation true if it sends all the orientation preserving/reversing elements of the fundamental group of $S$ into orientation preserving/reversing elements of the fundamental group of $\mathrm{R} P^{2}$. Otherwise we say that the element is non orientation true.

- We will denote by $\theta_{0}$ the trivial homomorphism, and by $\theta_{1}$ the (unique) homomorphism that send to -1 each orientation reversing element, and to 1 all the other elements. It is clear that $\theta_{1}$ corresponds to the orientation class $w_{1}(\tau S) \in H^{1}(S ; \mathbf{Z} / 2)$.

- We denote by $Z^{T}[x]$ the sheaf of local coefficients on $S$ defined by the class $x \in H^{1}(S ; \mathbf{Z} / 2)$.

- For any map $f: S \rightarrow \mathrm{R} P^{2}$, the (twisted) degree of $f$ is the integer $d(f)$ (possible mod 2) defined by $f^{*}(\mu)=d(f) v$, where $f^{*}$ is the induced homomorphism $f^{*}: H^{2}\left(\mathrm{R} P^{2} ; \mathbf{Z}^{T}\left[w_{1}\left(\tau \mathrm{R} P^{2}\right)\right]\right) \rightarrow H^{2}\left(S ; \mathbf{Z}^{T}\left[f^{*}\left(w_{1}\left(\tau \mathrm{R} P^{2}\right)\right)\right]\right)$ and $\mu$ and $v$ are the fundamental classes of $H^{2}\left(S ; \mathbf{Z}^{T}\left[w_{1}\left(\tau \mathrm{R} P^{2}\right)\right]\right)$ and $H^{2}\left(S ; \mathbf{Z}^{T}\left[f^{*}\left(w_{1}\left(\tau \mathrm{R} P^{2}\right)\right)\right]\right)$, respectively.

- The notation $[X, Y]$ is for the set of free homotopy classes of maps, $[X, Y]_{*}$ for the set of based homotopy classes of maps. The notation $[X, Y]_{*}^{\theta}$ means that any map in any class of this set induces the homomorphism $\theta$ on 
the fundamental group. In the case that $\pi_{1}(Y)=\mathrm{Z} / 2$ then the induced homomorphism $f_{\#}: \pi_{1}(X) \rightarrow \pi_{1}(Y)$ is independent of the base point, and we denote by $[X, Y]^{\theta}$ the set of homotopy classes of maps where any class of this set induces the homomorphism $\theta$ on the fundamental group.

- The notation $m(X, Y ; f)$ is for the component of the set of maps from $X$ to $Y$ that contains $f$.

We can enumerate the components of the space $\left(\mathrm{R} P^{2}\right)^{S}$ as follows.

Proposition 2.1. The set of components of $\left(\mathrm{R} P^{2}\right)^{S}$ is described as follows. If $S$ is oriented,

$$
\begin{aligned}
m\left(S, \mathrm{R} P^{2}\right)=\bigsqcup_{k=0}^{\infty} m\left(S, \mathrm{R} P^{2} ; f_{2 k}\right) \\
\sqcup \bigsqcup_{x \in H^{1}(S ; Z / 2), x \neq 0}\left(m\left(S, \mathrm{R} P^{2} ; f_{x}\right) \sqcup m\left(S, \mathrm{R} P^{2} ; f_{-x}\right)\right),
\end{aligned}
$$

where the homomorphism induced in the fundamental group and the corresponding class in $H^{1}$ are

$$
\begin{array}{ll}
\left(f_{2 k}\right)_{*}=\theta_{0}, & x=0 \\
\left(f_{ \pm x}\right)_{*}=\theta \neq \theta_{0}, & x \neq 0,
\end{array}
$$

and the twisted degree is

$$
d\left(f_{2 k}\right)=2 k, \quad d\left(f_{ \pm x}\right)=0 .
$$

If $S$ is nonorientable

$$
\begin{aligned}
m\left(S, \mathrm{R} P^{2}\right)= & \bigsqcup_{k=0}^{\infty} m\left(S, \mathrm{R} P^{2} ; f_{2 k+1}\right) \sqcup \bigsqcup_{x \in H^{1}(S ; \mathrm{Z} / 2), x \neq w_{1}(\tau S), x^{2}=1} m\left(S, \mathrm{R} P^{2} ; f_{x}^{1}\right) \\
& \sqcup \bigsqcup_{x \in H^{1}(S ; \mathrm{Z} / 2), x \neq w_{1}(\tau S), x^{2}=0}\left(m\left(S, \mathbf{R} P^{2} ; f_{x}^{0}\right) \sqcup m\left(S, \mathrm{R} P^{2} ; f_{-x}^{0}\right)\right),
\end{aligned}
$$

where

$$
\begin{aligned}
\left(f_{2 k+1}\right)_{*} & =\theta_{1}, & & x=w_{1}(\tau S) \\
\left(f_{ \pm x}^{i}\right)_{*} & =\theta \neq \theta_{1}, & & x \neq w_{1}(\tau S),
\end{aligned}
$$

and

$$
d\left(f_{2 k+1}\right)=2 k+1, \quad d\left(f_{ \pm x}^{i}\right)=i .
$$

Next, in order to apply the technique developed in [9] to compute the fundamental group of the space of sections of some bundles, we need some results 
about the Euler class of some plane bundles. First, we fix some further notation. In the following a section $s$ of a vector bundle is a nowhere vanishing section, and $\xi$ is a 3 dimensional vector bundle over a space $X$.

- For any trivial bundle $\xi$ over $X$, we will identify the space of sections $\Gamma(X, E S \xi)$ with the mapping space $m(X, F S \xi)$, where $E S \xi$ is the total space of the spherical bundle of $\xi$ and $F S \xi$ its fiber.

- $\xi$ has a section if and only if it is the summand of a trivial line bundle, namely there exists a 2-plane bundle $\eta$ such that $\xi=\eta \oplus 1$ (Withney sum).

- If the associated projective bundle $P \xi$ has a section, then there exist a line bundle $\lambda$ and a 2-plane bundle $\eta$ over $X$ such that $P \xi=\eta \oplus \lambda$.

- For any line bundle $\lambda, P \xi=P(\xi \otimes \lambda)$.

- If $\xi$ is trivial and $P \xi$ has a section $s$, then $\xi=s^{-1}\left(\tau \operatorname{R} P^{2} \oplus \gamma\right)$, where $\gamma$ is the Hopf line bundle and $\tau \mathrm{R} P^{2}$ is the tangent bundle over $\mathrm{R} P^{2}$.

Lemma 2.2. If $\xi$ is trivial and $P \xi$ has a section $s$, then $\xi \otimes s^{-1} \gamma=$ $s^{-1} \tau \mathrm{R} P^{2} \oplus 1$.

Proof. If $P \xi$ has a section, then $\xi=s^{-1}\left(\tau \mathrm{R} P^{2} \oplus \gamma\right)$. The thesis follows since $\tau \mathrm{R} P^{2}=\tau \mathrm{R} P^{2} \otimes \gamma$ by [1].

Lemma 2.3. Let $f: S \rightarrow \mathrm{R} P^{2}$, and let $\eta=f^{-1} \tau \mathrm{R} P^{2}$. Then the Euler class of $\eta$ is determined by the degree of $f$, while the first Stiefel Whitney class of $\eta$ is determined by the homomorphism induced by $f$ on the fundamental group, namely

$$
\begin{aligned}
\chi(\eta) & =d(f), \\
w_{1}(\eta) & =f^{*}\left(w_{1}\left(\tau \mathrm{R} P^{2}\right)\right) .
\end{aligned}
$$

Note that, if $f^{*}\left(w_{1}\left(\tau \mathrm{R} P^{2}\right)\right)=x \neq w_{1}(\tau S)$, then

$$
\chi(\eta)=d(f)=\left\{\begin{array}{ll}
0, & w_{1}(\tau S)=0, \\
x^{2}, & w_{1}(\tau S) \neq 0 .
\end{array} .\right.
$$

We recall now two results of [9], that will be used to prove our main theorems in Sections 3 and 4. It should be observed that we can not apply Theorems 1, 2 or 3 of [9] directely except for the case of the component of the constant map. We prefer to recall here the complete statement of these theorems using our notation, in order to make easier to understand the calculations of the following sections.

THEOREM 2.4 (Larmore-Thomas). Let $s^{\prime}$ be a given section of a 3-plane bundle $\xi^{\prime}$ over $S$, and $\Gamma^{\prime}=\Gamma\left(S, E S \xi^{\prime} ; s^{\prime}\right)$. Then, $\xi^{\prime}=\eta^{\prime} \oplus 1$, for some 2-plane 
bundle $\eta^{\prime}$, and we have an exact sequence

$$
H^{0}\left(S ; \mathbf{Z}^{T}\left[w_{1}\left(\eta^{\prime}\right)\right]\right) \stackrel{\cup x\left(\eta^{\prime}\right)}{\longrightarrow} H^{2}(S ; \mathbf{Z}) \stackrel{\phi}{\rightarrow} \pi_{1}\left(\Gamma^{\prime}\right) \stackrel{p}{\rightarrow} H^{1}\left(S ; \mathbf{Z}^{T}\left[w_{1}\left(\eta^{\prime}\right)\right]\right) \rightarrow 0 .
$$

The following information is sufficient to determine $\pi_{1}\left(\Gamma^{\prime}\right)$ as an extension:

(1) $\phi\left(H^{2}(S ; Z)\right)$ is central.

(2) If $\alpha_{1}, \alpha_{2} \in \pi_{1}\left(\Gamma^{\prime}\right)$ and $p\left(\alpha_{1}\right)=x_{1}, p\left(\alpha_{2}\right)=x_{2}$, then $\left[\alpha_{1}, \alpha_{2}\right]=$ $\phi\left(2 x_{1} \cup x_{2}\right)$.

(3) If $w_{1}\left(\eta^{\prime}\right) \neq 0$, let $\bar{w}_{1}$ be the unique element of $H^{1}\left(S ; Z^{T}\left[w_{1}\left(\eta^{\prime}\right)\right]\right)$ of order 2. Chose $z \in H^{2}(S ; Z)$ such that $z \bmod 2=w_{2}\left(\eta^{\prime}\right)$. Then there exists $y \in \pi_{1}\left(\Gamma^{\prime}\right)$ such that $p(y)=\bar{w}_{1}$ and $y^{2}=\phi\left(\bar{w}_{1} \cup \bar{w}_{1}+z\right)$.

Note that in the next theorem we will identify the elements of $\pi_{1}\left(\Gamma^{\prime}\right)$ with their images in $\pi_{1}(\Gamma)$ when there is no ambiguity.

THeOREM 2.5 (Larmore-Thomas). Let s be a given section of the projective bundle $P \xi$ associated to a 3-plane bundle $\xi$ over $S$, and assume that $s$ is the identification of a section $s^{\prime}$ of an associated sphere bundle $S \xi^{\prime}$ such that $P \xi^{\prime}=$ $P \xi$. Let $\Gamma=\Gamma(S, E P \xi ; s)$, and $\Gamma^{\prime}=\Gamma\left(S, E S \xi^{\prime} ; s^{\prime}\right)$. Then, $\xi^{\prime}=\eta^{\prime} \oplus 1$, for some 2-plane bundle $\eta^{\prime}$, we have a diagram with exact row and column

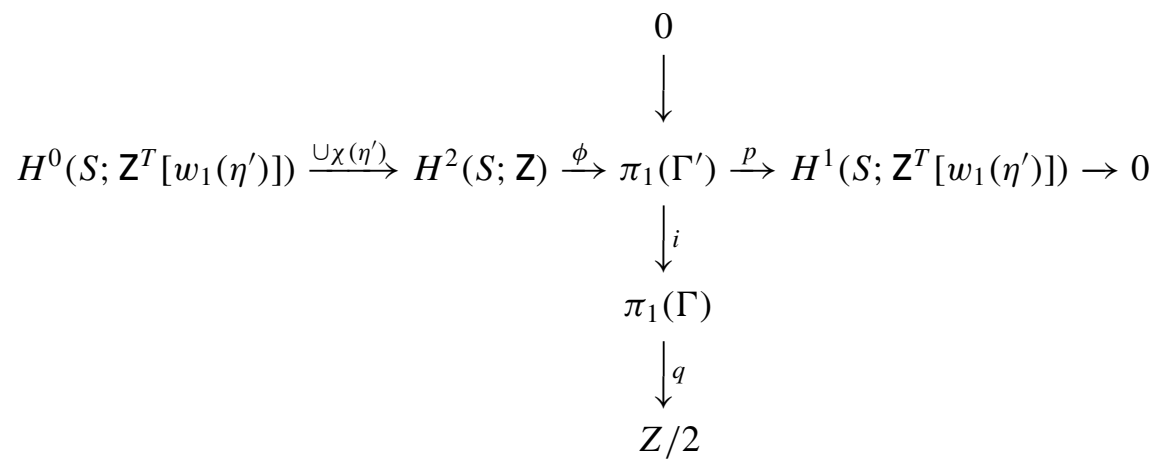

and the following information determines $\pi_{1}(\Gamma)$ :

(1) $\pi_{1}\left(\Gamma^{\prime}\right)$ is given in Theorem 2.4 .

(2) $\chi\left(\eta^{\prime}\right) \neq 0$ if and only if $\pi_{1}(\Gamma)=\pi_{1}\left(\Gamma^{\prime}\right)$.

(3) When $\chi\left(\eta^{\prime}\right)=0$, the following conditions apply:

(a) $i \phi\left(H^{2}(S ; \mathrm{Z})\right)$ is central.

(b) There exists an element $t \in \pi_{1}(\Gamma)$ (defined by the section due to the splitting $\left.\eta^{\prime}=\zeta^{\prime} \oplus 1\right)$ such that $q(t)=1$.

(c) For each $x \in H^{1}\left(S ; Z^{T}\left[w_{1}\left(\eta^{\prime}\right)\right]\right)$, there exists a $g \in \pi_{1}\left(\Gamma^{\prime}\right)$ such that $p(g)=x$ and $\operatorname{tgt}^{-1} g=\phi(x \cup x)$. 
(d) If $w_{1}\left(\eta^{\prime}\right)=0$, then $t^{2}=1$.

(e) If $w_{1}\left(\eta^{\prime}\right) \neq 0$, then $p\left(t^{2}\right)=\bar{w}_{1}$ and $t^{2}=y$ (the element defined in (3) of Theorem 2.4).

In order to compute the fundamental group of the components of $\left(\mathrm{R} P^{2}\right)^{S}$, we will follow the routine described below.

(1) Fix $\xi$ to be the trivial 3-plane bundle over $S$. Then, we have the bijection $m\left(S, \mathrm{R} P^{2} ; f_{s}\right)=\Gamma\left(S, P \xi ; s_{f}\right)$ (note that we can apply here Theorem 3 of [9] only if we are dealing with the trivial/constant section; in fact, we are concerned with splittings arising from generic sections of the projective bundle)

(2) Let $s$ be a fixed section of $P \xi$. Then, $\xi=s^{-1} \tau \mathrm{R} P^{2} \oplus s^{-1} \gamma$, and $\xi^{\prime}=$ $\xi \otimes s^{-1} \gamma=3 s^{-1} \gamma=s^{-1} \tau \mathrm{R} P^{2} \oplus 1$ (again we can not apply Theorem 3 [9], since the splitting of $\xi$ is not with a trivial line bundle).

(3) Since $\xi^{\prime}=s^{-1} \tau \mathrm{R} P^{2} \oplus 1$, there exists a section of the spherical bundle $s^{\prime}: S \rightarrow S \xi^{\prime}$, that covers $s$, as $S \xi$ covers $P \xi$.

(4) This means that we can compute $\pi_{1}\left(\Gamma\left(S, E S \xi^{\prime} ; s^{\prime}\right)\right)$, using Theorem 2.4 for the spherical cases (note that we can not use Theorems 1 or 2 of [9], since $\xi^{\prime}$ is nonorientable).

(5) Next, we can use Theorem 2.5 to compute $\pi_{1}(\Gamma(S, E P \xi ; s)$.

Note that the 2-plane bundles $\eta$ and $\eta^{\prime}$ appearing in the splitting of the spherical and projective bundle given in Theorems 2.4 and 2.5, are in fact the same bundle: $\eta^{\prime}=\eta=f_{s}^{-1} \tau \mathrm{R} P^{2}$. This is the main point in the following computations.

We conclude this section, by recalling some results about cohomology and cup product on surfaces. If $S$ is oriented, it is the sum of $g$ tori. Then, we can assume that there exists a set of generators $\left\{a_{1}, b_{1}, \ldots, a_{g}, b_{g}\right\}$ for $H^{1}(S ; \mathbf{Z})$, satisfying the following rules

$$
a_{i} \cup b_{j \neq i}=a_{i} \cup a_{j}=b_{i} \cup b_{j}=0, \quad a_{i} \cup b_{i}=\Lambda,
$$

where $\Lambda$ is a fixed generator for $H^{2}(S ; Z)$. Similarly, if $S$ is nonorientable, it is a sum of $g$ projective spaces, and we take a set of generators $\left\{c_{1}, \ldots, c_{g}\right\}$ for $H^{1}(S ; \mathbf{Z} / 2)$, such that

$$
c_{i} \cup c_{j \neq i}=0, \quad c_{i} \cup c_{i}=\Lambda .
$$

Note that the orientation class is $w_{1}(\tau S)=c_{1}+\cdots+c_{g}$. 


\section{Nonorientable surfaces}

In this section we compute the fundamental group of the different components of $m\left(S, \mathrm{R} P^{2}\right)$ when $S$ is nonorientable. We decompose the result using the enumeration of the components provided by Proposition 2.1 and we proceed following the routine presented in Section 2. In all cases, the 2-plane bundle $\eta=\eta^{\prime}$ is the pullback bundle $s^{-1} \tau \mathrm{R} P^{2}$ of the tangent bundle over R $P^{2}$ induced by a map in the mapping space under study.

In order to state our result, we introduce the following notation. Let $\mathscr{B}_{l, m}$ be the group with generators $\gamma_{1}, \ldots, \gamma_{l}, \lambda, t$ and relations

$$
\begin{aligned}
t^{m} & =1, \\
\lambda^{2} & =1, \\
t \gamma_{j} t^{-1} \gamma_{j} & =\lambda, \\
{\left[\gamma_{j}, \gamma_{k}\right]=\left[\lambda, \gamma_{j}\right]=[t, \lambda] } & =1 .
\end{aligned}
$$

Then we have the following theorem, where a more precise description of the group's generators is given in the course of the proof.

THEOREM 3.1. If $S$ is a nonorientable surface homeomorphic to the connected sum of $g$ copies of $\mathrm{R} P^{2}$, then

$$
\begin{gathered}
\pi_{1}\left(m\left(S, \mathrm{R} P^{2} ; f_{2 k+1}\right)\right)=\left\{\begin{array}{l}
\mathbf{Z}^{g-1} \oplus \mathrm{Z} / 2, \quad \text { if } g \text { odd }, \\
\mathbf{Z}^{g-1} \oplus \mathrm{Z} / 4, \text { if } g \text { even }
\end{array}\right. \\
\pi_{1}\left(m\left(S, \mathbf{R} P^{2} ; f_{x}^{1}\right)\right)=\mathbf{Z}^{g-2} \oplus \mathbf{Z} / 2 \oplus \mathbf{Z} / 2, \\
\pi_{1}\left(m\left(S, \mathbf{R} P^{2} ; f_{ \pm x}^{0}\right)\right)= \begin{cases}\mathscr{B}_{g-1,2}, & \text { if } x=0, \\
\mathscr{B}_{g-2,4}, & \text { if } x \neq 0 .\end{cases}
\end{gathered}
$$

Proof. We split the proof in three parts, corresponding to the decomposition given in Proposition 2.1.

Part 1. $\pi_{1}\left(m\left(S, R P^{2} ; f_{2 k+1}\right)\right)=\pi_{1}\left(\Gamma\left(S, E P \xi ; f_{2 k+1}\right)\right)$. By Proposition 2.1 and Lemma 2.3, the Euler class is

$$
\chi\left(\eta^{\prime}\right)=\chi(\eta)=d\left(f_{2 k+1}\right)=2 k+1 \neq 0,
$$

while the first SW class is

$$
w_{1}\left(\eta^{\prime}\right)=w_{1}(\eta)=f_{2 k+1}^{*}\left(w_{1}\left(\tau \mathrm{R} P^{2}\right)\right)=\theta_{1}\left(w_{1}\left(\tau \mathrm{R} P^{2}\right)\right)=w_{1}(\tau S) .
$$


By (2) of Theorem 2.5, since $\chi\left(\eta^{\prime}\right) \neq 0$, we have that

$$
\pi_{1}\left(\Gamma\left(S, E P \xi ; f_{2 k+1}\right)\right)=\pi_{1}\left(\Gamma\left(S, E S \xi^{\prime} ; f_{2 k+1}^{\prime}\right)\right),
$$

so we use Theorem 2.4 in order to compute $\pi_{1}(\Gamma)=\pi_{1}\left(\Gamma^{\prime}\right)=\pi_{1}\left(\Gamma\left(S, E S \xi^{\prime}\right.\right.$; $\left.f_{2 k+1}^{\prime}\right)$ ). Before, we need the following fact about cup product. For any $x \in$ $H^{1}(S ; \mathrm{Z} / 2)$, we have the commutative diagram

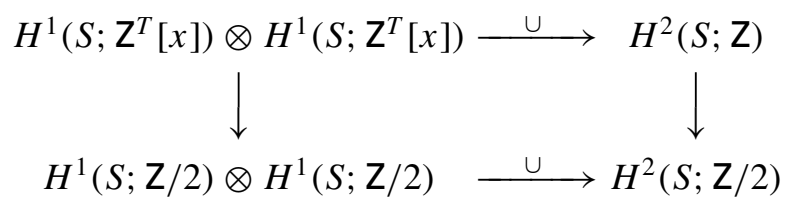

where the vertical arrows are reduction mod 2. Since in the present case $S$ is nonorientable, we have isomorphism $H^{2}(S ; \mathbf{Z})=H^{2}(S ; \mathbf{Z} / 2)$. So therefore, we can use mod 2 cohomological cup product in order to find out if some twisted coefficient cup product is non vanishing.

Since $w_{1}\left(\eta^{\prime}\right)=w_{1}(\tau S)$, and since, by duality $H^{1}\left(S ; Z^{T}\left[w_{1}(\tau S)\right]\right)=$ $H_{1}(S ; \mathrm{Z})$, by Theorem 2.4, we have the exact sequence

$$
0 \longrightarrow \mathrm{Z} / 2[\Lambda] \stackrel{\phi}{\longrightarrow} \pi_{1}\left(\Gamma^{\prime}\right) \stackrel{p}{\longrightarrow} \mathrm{Z}\left[\bar{c}_{1}, \ldots, \bar{c}_{g-1}\right] \oplus \mathrm{Z} / 2\left[\bar{w}_{1}(\tau S)\right] \longrightarrow 0,
$$

where the bar notation is for the classes in $H^{1}\left(S ; \mathbf{Z}^{T}\left[w_{1}(\tau S)\right]\right)$ that reduce modulo 2 to the respective classes in $H^{1}(S ; Z / 2)$. See also the remark at page 235 of [9]. In order to find the extension, we start with a set of generators

$$
\left\{\gamma_{1}, \ldots, \gamma_{g-1}, \delta, \lambda\right\}
$$

such that

$$
\begin{aligned}
p\left(\gamma_{i}\right) & =\bar{c}_{i}, \\
p(\delta) & =\bar{w}_{1}(\tau S), \\
\lambda & =\phi(\Lambda) .
\end{aligned}
$$

Now, by (1) of Theorem $2.4, \lambda$ is central. Also, $\lambda^{2}=\phi(2 \Lambda)=\phi(0)=1$. By (2) of the same theorem, $\left[\gamma_{j}, \gamma_{k}\right]=\left[\gamma_{j}, \delta\right]=0$, since the cup product of each two elements has order 2. This means that the extension is abelian. Therefore, the only problem is to determine its torsion component. The torsion can only be $\mathbf{Z} / 2 \oplus \mathbf{Z} / 2$ or $\mathbf{Z} / 4$. By (3) of Theorem 2.4 , there exists an element $y \in \pi_{1}\left(\Gamma^{\prime}\right)$, such that

$$
p(y)=\bar{w}_{1}(\tau S), \quad y^{2}=\phi\left(\bar{w}_{1}(\tau S) \cup \bar{w}_{1}(\tau S)+z\right),
$$

where $z \in H^{2}\left(S ;\right.$ Z) with $z \bmod 2=w_{2}(\eta)$. Since $w_{2}(\eta)=\chi\left(\eta^{\prime}\right) \bmod 2=$ $(2 k+1) \bmod 2=1 \neq 0$, clearly $z=\Lambda \neq 0$. Since, $\bar{w}_{1}(\tau S) \bmod 2=$ 
$w_{1}(\tau S)=c_{1}+\cdots+c_{g}$, and $w_{1}(\tau S) \cup w_{1}(\tau S)=g \Lambda$, the above remark on the twisted cup product implies that $\bar{w}_{1}(\tau S) \cup \bar{w}_{1}(\tau S)=g \Lambda$. Therefore, $y^{2}=\phi\left(\bar{w}_{1}(\tau S) \cup \bar{w}_{1}(\tau S)+\Lambda\right)=\phi((g+1) \Lambda)=\lambda^{(g+1) \bmod 2}$, depends on the parity of $g$.

Note that, since $p(y)=\bar{w}_{1}(\tau S)$, it follows that $y=\lambda$ or $y=\delta \lambda$, and in both cases $y^{2}=\delta^{2}$. Therefore, up to a change of the generator $\delta$ into $\delta \lambda$, we can always assume $y=\lambda$.

We have the two possibilities.

(1) If $g$ is odd, then $\delta^{2}=1$, therefore we have two generators of order 2, and the extension is $\pi_{1}(\Gamma)=\mathrm{Z}\left[\gamma_{1}, \ldots, \gamma_{g-1}\right] \oplus \mathbf{Z} / 2[\delta, \lambda]$.

(2) If $g$ is even, then $\delta^{2}=\lambda$. This means that in this case the torsion component is $\mathbf{Z} / 4$ and $\pi_{1}(\Gamma)=\mathbf{Z}\left[\gamma_{1}, \ldots, \gamma_{g-1}\right] \oplus \mathbf{Z} / 4[\delta]$.

Part 2. $\pi_{1}\left(m\left(S, \mathrm{R} P^{2} ; f_{x}^{1}\right)\right)=\pi_{1}\left(\Gamma\left(S, E P \xi ; f_{x}^{1}\right)\right.$. Now, the Euler class is $\chi\left(\eta^{\prime}\right)=\chi(\eta)=d\left(f_{x}^{1}\right) \neq 0$, while the first SW class is $w_{1}\left(\eta^{\prime}\right)=w_{1}(\eta)=$ $\left(f_{x}^{1}\right)^{*}\left(w_{1}\left(\tau \mathrm{R} P^{2}\right)\right)=\theta\left(w_{1}\left(\tau \mathrm{R} P^{2}\right)\right)=x \neq w_{1}(\tau S), 0$, since $\theta \neq \theta_{1}$, and since $x \cup x \neq 0$.

As before, by (2) of Theorem 2.5, since $\chi\left(\eta^{\prime}\right) \neq 0$, we have that

$$
\pi_{1}\left(\Gamma\left(S, E P \xi ; f_{x}^{1}\right)\right)=\pi_{1}\left(\Gamma\left(S, E S \xi^{\prime} ;\left(f_{x}^{1}\right)^{\prime}\right)\right),
$$

so we use Theorem 2.4 in order to compute $\pi_{1}(\Gamma)=\pi_{1}\left(\Gamma^{\prime}\right)=\pi_{1}\left(\Gamma\left(S, E S \xi^{\prime}\right.\right.$; $\left.\left.\left(f_{x}^{1}\right)^{\prime}\right)\right)$. We have the exact sequence

$$
H^{0}\left(S ; \mathbf{Z}^{T}[x]\right) \longrightarrow H^{2}(S ; \mathbf{Z}) \stackrel{\phi}{\longrightarrow} \pi_{1}(\Gamma) \stackrel{p}{\longrightarrow} H^{1}\left(S ; \mathbf{Z}^{T}[x]\right) \longrightarrow 0,
$$

that since $x \neq 0$, becomes

$$
0 \longrightarrow \mathrm{Z} / 2[\Lambda] \stackrel{\phi}{\longrightarrow} \pi_{1}\left(\Gamma^{\prime}\right) \stackrel{p}{\longrightarrow} \mathrm{Z}\left[\bar{c}_{1}, \ldots, \bar{c}_{g-2}\right] \oplus \mathbf{Z} / 2[\bar{x}] \longrightarrow 0 .
$$

The situation is very much the same as in previous Part 1 , with the class $\bar{x}$ instead of $\bar{w}_{1}(\tau S)$. We take a set of generators

$$
\left\{\gamma_{1}, \ldots, \gamma_{g-1}, \delta, \lambda\right\}
$$

such that

$$
\begin{aligned}
p\left(\gamma_{i}\right) & =\bar{c}_{i}, \\
p(\delta) & =\bar{x}, \\
\lambda & =\phi(\Lambda) .
\end{aligned}
$$

By (1) of Theorem 2.4, $\lambda$ is central. Also, $\lambda^{2}=\phi(2 \Lambda)=\phi(0)=1$. By (2) of the same theorem, $\left[\gamma_{j}, \gamma_{k}\right]=\left[\gamma_{j}, \delta\right]=0$, since the cup product of each two elements has order 2 . Thus the extension is abelian and the torsion can only be 
$\mathbf{Z} / 2 \oplus \mathbf{Z} / 2$ or $\mathbf{Z} / 4$. By (3) of Theorem 2.4 , there exists an element $y \in \pi_{1}\left(\Gamma^{\prime}\right)$, such that

$$
p(y)=\bar{x}, \quad y^{2}=\phi(\bar{x} \cup \bar{x}+z),
$$

where $z=\Lambda \in H^{2}(S ; \mathbf{Z})$, since $z \bmod 2=w_{2}(\eta)$, and $\chi(\eta)=1$. Also, now $x \cup x \neq 0$, and therefore $\bar{x} \cup \bar{x}=\Lambda$, and $y^{2}=\phi(2 \Lambda)=\phi(0)=1$. Again, by changing the generators, we can assume $y=\delta$. Thus, $\delta^{2}=1$, i.e. $\delta$ is a generator of order 2 , and the extension is $\pi_{1}(\Gamma)=\mathrm{Z}\left[\gamma_{1}, \ldots, \gamma_{g-2}\right] \oplus \mathrm{Z} / 2[\delta, \lambda]$.

Part 3. $\pi_{1}\left(m\left(S, \mathrm{R} P^{2} ; f_{ \pm x}^{0}\right)\right)=\pi_{1}\left(\Gamma\left(S, E P \xi ; f_{ \pm x}^{0}\right)\right.$. Now, we have that $\chi\left(\eta^{\prime}\right)=\chi(\eta)=d\left(f_{ \pm x}^{0}\right)=0$, while $w_{1}\left(\eta^{\prime}\right)=w_{1}(\eta)=\left(\left(f_{ \pm x}^{0}\right)^{*}\left(w_{1}\left(\tau \mathbf{R} P^{2}\right)\right)=\right.$ $\theta\left(w_{1}\left(\tau \operatorname{R} P^{2}\right)\right)=x \neq w_{1}(\tau S)$, since $\theta \neq \theta_{1}$. Recall that $x \cup x=0$ in the present case.

By (2) of Theorem 2.5, since $\chi\left(\eta^{\prime}\right)=0$, we have that

$$
\pi_{1}(\Gamma)=\pi_{1}\left(\Gamma\left(S, E P \xi ; f_{x}^{1}\right)\right) \neq \pi_{1}\left(\Gamma^{\prime}\right)=\pi_{1}\left(\Gamma\left(S, E S \xi^{\prime} ;\left(f_{x}^{1}\right)^{\prime}\right)\right),
$$

but can be determined using the extension given in Theorem 2.5. We distinguish between the two cases $x=0, x \neq 0$.

Case 3.1. $x=0$. First, we use Theorem 2.4 in order to compute $\pi_{1}\left(\Gamma^{\prime}\right)$. Since $x=0$, we have the exact sequence

$$
\mathrm{Z} \stackrel{0}{\longrightarrow} \mathrm{Z} / 2[\Lambda] \stackrel{\phi}{\longrightarrow} \pi_{1}\left(\Gamma^{\prime}\right) \stackrel{p}{\longrightarrow} \mathrm{Z}\left[\bar{c}_{1}, \ldots, \bar{c}_{g-1}\right] \longrightarrow 0 .
$$

In order to find the extension, we take the set of generators

$$
\left\{\gamma_{1}, \ldots, \gamma_{g-1}, \lambda\right\}
$$

such that

$$
\begin{aligned}
p\left(\gamma_{i}\right) & =c_{i}, \\
\lambda & =\phi(\Lambda) .
\end{aligned}
$$

Now, by (1) of Theorem 2.4, $\lambda$ is central. By (2) of the same theorem, $\left[\gamma_{j}, \gamma_{k}\right]=0$. This means that the extension is abelian. Since the unique torsion element is the image of the generator of $Z / 2$, we have that $\pi_{1}\left(\Gamma^{\prime}\right)=$ $\mathrm{Z}\left[\gamma_{1}, \ldots, \gamma_{g-1}\right] \oplus \mathrm{Z} / 2[\lambda]$. 
Second, we use the exact sequence given in Theorem 2.5.

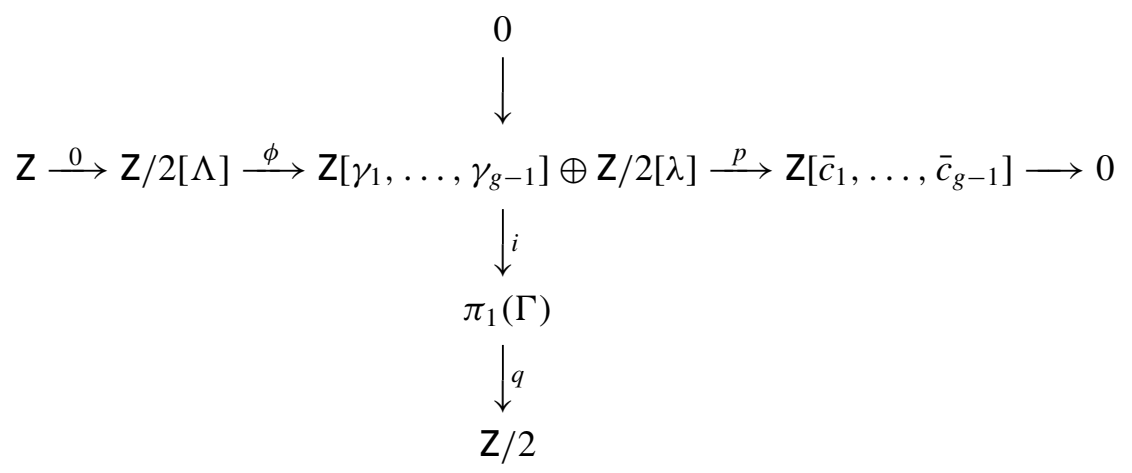

Consider the set of generators

$$
\left\{\hat{\gamma}_{1}, \ldots, \hat{\gamma}_{g-1}, \hat{\lambda}, t\right\}
$$

such that

$$
\begin{aligned}
\hat{\gamma}_{j} & =i\left(\gamma_{j}\right), \\
\hat{\lambda} & =i(\lambda), \\
q(t) & =1 .
\end{aligned}
$$

We have the following facts:

- since $w_{1}(\eta)=x=0$, by (3d) of Theorem 2.5, $t^{2}=1$;

- $\hat{\lambda}^{2}=i(2 \lambda)=i(0)=1$;

- all the generators $\hat{\gamma}_{j}$ and $\hat{\lambda}$ commute with each others, since they are in the image of $i$;

- by (3a) of Theorem $2.5, \hat{\lambda}$ is central, and therefore $[\hat{\lambda}, t]=1$.

- by (3c) of Theorem 2.5, since $\hat{\gamma}_{j}=i\left(\gamma_{j}\right)$ and $p\left(\gamma_{j}\right)=\bar{c}_{j}$,

$$
t \hat{\gamma}_{j} t \hat{\gamma}_{j}=i \phi\left(\bar{c}_{j} \cup \bar{c}_{j}\right)=i \phi(\Lambda)=i(\lambda)=\hat{\lambda} .
$$

Thus, we have proved that (where we identify the elements of $\pi_{1}\left(\Gamma^{\prime}\right)$ with their images in $\pi_{1}(\Gamma)$ )

$$
\begin{aligned}
& \pi_{1}(\Gamma)=\left\langle\gamma_{1}, \ldots, \gamma_{g-1}, \lambda, t\right. \\
& \left.\left[\gamma_{j}, \gamma_{k}\right]=\left[\gamma_{j}, \lambda\right]=[t, \lambda]=1, \lambda^{2}=t^{2}=1, t \gamma_{j} t \gamma_{j}=\lambda\right\rangle .
\end{aligned}
$$

Case 3.2. $x \neq 0$. Now by Theorem 2.4, since $x \neq 0, w_{1}(\tau S)$, we have the exact sequence

$$
0 \longrightarrow \mathbf{Z} / 2[\Lambda] \stackrel{\phi}{\longrightarrow} \pi_{1}\left(\Gamma^{\prime}\right) \stackrel{p}{\longrightarrow} \mathbf{Z}\left[\bar{c}_{1}, \ldots, \bar{c}_{g-2}\right] \oplus \mathbf{Z} / 2[\bar{x}] \longrightarrow 0 .
$$


Thus we are precisely in the same situation as in the Part 2, with the unique difference that here both $\chi(\eta)$ and $x \cup x$ vanish. Thus, the extension is abelian and we just need to determine the torsion component. The element $y \in \pi_{1}\left(\Gamma^{\prime}\right)$ required by (3) of Theorem 2.4 satisfies the conditions

$$
p(y)=\bar{x}, \quad y^{2}=\phi(\bar{x} \cup \bar{x}+z),
$$

with $z \bmod 2=w_{2}(\eta)=0$ and $\bar{x} \cup \bar{x}=0$. Thus, $y^{2}=\phi(0)=1$, and the extension is $\pi_{1}(\Gamma)=\mathbf{Z}\left[\gamma_{1}, \ldots, \gamma_{g-2}\right] \oplus \mathbf{Z} / 2[\delta, \lambda]$.

Next, we use the exact sequence given in Theorem 2.5.

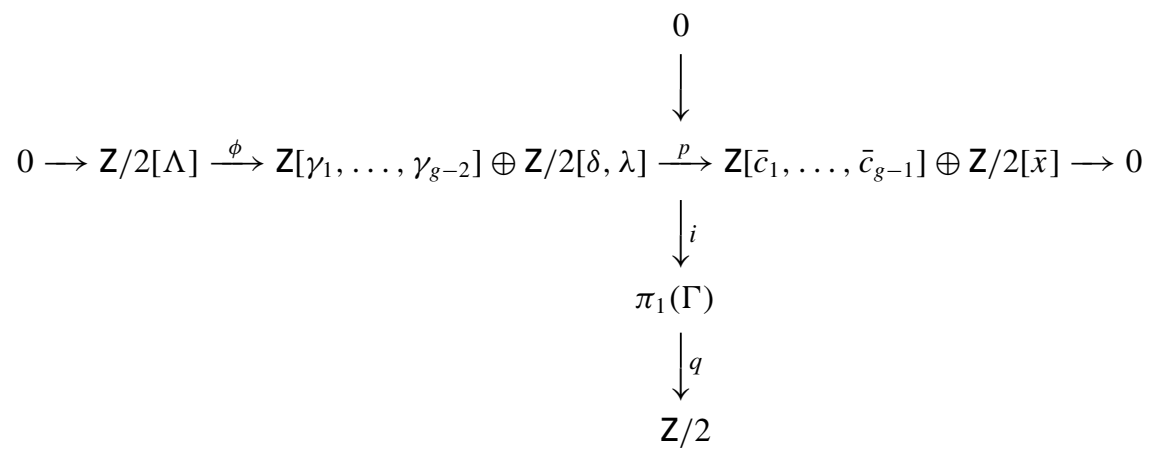

Consider the set of generators

$$
\left\{\hat{\gamma}_{1}, \ldots, \hat{\gamma}_{g-2}, \hat{\delta}, \hat{\lambda}, t\right\}
$$

such that

$$
\begin{aligned}
\hat{\gamma}_{j} & =i\left(\gamma_{j}\right), \\
\hat{\delta} & =i(\delta), \\
\hat{\lambda} & =i(\lambda), \\
q(t) & =1 .
\end{aligned}
$$

We have the following facts:

- since $w_{1}(\eta)=x \neq 0$, by (3e) of Theorem $2.5, t^{2}=\hat{\delta}$;

- $\hat{\delta}^{2}=i(2 \delta)=i(0)=1, \hat{\lambda}^{2}=i(2 \lambda)=i(0)=1$; so $t^{4}=1$;

- all the generators $\hat{\gamma}_{j}, \hat{\delta}$, and $\hat{\lambda}$ commute with each others, since they are in the image of $i$;

- by (3a) of Theorem $2.5, \hat{\lambda}$ is central;

- by (3c) of Theorem 2.5, since $\hat{\gamma}_{j}=i\left(\gamma_{j}\right)$ and $p\left(\gamma_{j}\right)=\bar{c}_{j}$,

$$
t \hat{\gamma}_{j} t^{-1} \hat{\gamma}_{j}=i \phi\left(\bar{c}_{j} \cup \bar{c}_{j}\right)=i \phi(\Lambda)=i(\lambda)=\hat{\lambda} .
$$


Thus, we have proved that

$$
\begin{aligned}
& \pi_{1}(\Gamma)=\left\langle\gamma_{1}, \ldots, \gamma_{g-2}, \lambda, t\right. \\
& \left.\lambda^{2}=t^{4}=1, t \gamma_{j} t^{-1} \gamma_{j}=\lambda,\left[\gamma_{j}, \gamma_{k}\right]=\left[\gamma_{j}, \lambda\right]=[t, \lambda]=1\right\rangle .
\end{aligned}
$$

REMARK 3.2. As observed in the introduction, the case of the component of the identity map of $\left(\mathrm{R} P^{2}\right)^{\mathrm{R} P^{2}}$ can be tackled using a result of [13]. In fact, by Theorem 4.3 of [13]

$$
\pi_{1}\left(m\left(\mathrm{R} P^{2}, \mathrm{R} P^{2} ; \mathrm{id}\right)\right)=\mathrm{Z} / 2 \times \pi_{1}\left(m_{*}\left(\mathrm{R} P^{2}, \mathrm{R} P^{2} ; \mathrm{id}\right) / S O_{2}\right),
$$

and by Lemma 4.2 of the same work,

$$
\pi_{1}\left(m_{*}\left(\mathrm{R} P^{2}, \mathrm{R} P^{2} ; \mathrm{id}\right)\right)=\mathrm{Z} \times \pi_{1}\left(m_{*}\left(\mathrm{R} P^{2}, \mathrm{R} P^{2} ; \mathrm{id}\right) / S O_{2}\right) .
$$

We will show that $\pi_{1}\left(m_{*}\left(\mathrm{R} P^{2}, \mathrm{R} P^{2} ; \mathrm{id}\right)\right)$ is cyclic.

Therefore $\pi_{1}\left(m_{*}\left(\mathrm{R} P^{2}, \mathrm{R} P^{2} ; \mathrm{id}\right) / S O_{2}\right)=0$ and $\pi_{1}\left(m\left(\mathrm{R} P^{2}, \mathrm{R} P^{2} ; \mathrm{id}\right)\right)=$ $\mathrm{Z} / 2$. For, starting with the cofiber sequence

$$
\text { in }: \mathrm{R} P^{1} \rightarrow \mathrm{R} P^{2} \rightarrow S^{2},
$$

we obtain the fibration

$$
i n_{\#}: m_{*}\left(\mathrm{R} P^{2}, \mathrm{R} P^{2} ; \mathrm{id}\right) \rightarrow m_{*}\left(\mathrm{R} P^{1}, \mathrm{R} P^{2} ; i n\right),
$$

with fiber $\Omega_{i n}^{2} \mathrm{R} P^{2} \sim \Omega_{0}^{2} \mathrm{R} P^{2}$. Therefore, the associated long homotopy sequence reads as

$$
\begin{aligned}
\ldots \rightarrow \mathrm{Z} \rightarrow \mathrm{Z} \rightarrow \pi_{1}\left(m_{*}\left(\mathrm{R} P^{2}, \mathrm{R} P^{2} ; \mathrm{id}\right)\right) \\
\quad \rightarrow \pi_{2}\left(\mathrm{R} P^{2}\right)=\mathrm{Z} \rightarrow \pi_{2}\left(\mathrm{R} P^{2}\right)=\mathrm{Z} \rightarrow *,
\end{aligned}
$$

since $m_{*}\left(\mathrm{R} P^{2}, \mathrm{R} P^{2} ; \mathrm{id}\right)$ is connected. This proves that $\pi_{1}\left(m_{*}\left(\mathrm{R} P^{2}, \mathrm{R} P^{2} ; \mathrm{id}\right)\right)$ is at most $\mathbf{Z}$.

\section{Oriented surfaces with positive genus}

In this section we compute the fundamental group of the different components of $m\left(S, \mathrm{R} P^{2}\right)$ when $S$ is oriented and has genus $g>0$. We decompose the result using the enumeration of the components provided by Proposition 2.1 and we proceed following the routine presented in Section 2. In all cases, the 2-plane bundle $\eta=\eta^{\prime}$ is the pullback bundle $s^{-1} \tau \mathrm{R} P^{2}$ of the tangent bundle over $\mathrm{R} P^{2}$ induced by a map in the mapping space under study. 
In order to state our result, we introduce the following notation. Let $\mathscr{D}_{l, m, n}$ be the group with generators $\alpha_{1}, \beta_{1}, \ldots, \alpha_{l}, \beta_{l}, \lambda, t$ and relations

$$
\begin{aligned}
t^{m} & =1, \\
\lambda^{n} & =1, \\
t \alpha_{j} t^{-1} \alpha_{j}=t \beta_{j} t^{-1} \beta_{j} & =1, \\
{\left[\alpha_{j}, \alpha_{k}\right]=\left[\beta_{j}, \beta_{k}\right]=\left[\alpha_{j}, \beta_{k \neq j}\right] } & =1, \\
{\left[\alpha_{j}, \lambda\right]=\left[\beta_{j}, \lambda\right]=[t, \lambda] } & =1, \\
{\left[\alpha_{j}, \beta_{j}\right] } & =\lambda^{2} .
\end{aligned}
$$

Then we have the following theorem, where a more precise description of the group's generators is given in the course of the proof.

THEOREM 4.1. If $S$ is an oriented surface homeomorphic to the connected sum of $g \geq 1$ copies of $T$, then

$$
\begin{aligned}
& \pi_{1}\left(m\left(S, \mathrm{R} P^{2} ; f_{2 k}\right)\right)= \begin{cases}\mathscr{D}_{g, 1,2 k}, & \text { if } k>0, \\
\mathscr{D}_{g, 2, \infty}, & \text { if } k=0,\end{cases} \\
& \pi_{1}\left(m\left(S, \mathrm{R} P^{2} ; f_{ \pm x}\right)\right)=\mathscr{D}_{g-1,4, \infty} .
\end{aligned}
$$

Proof. We split the proof in 2 parts, corresponding to the decomposition given in Proposition 2.1.

Part 1. $\pi_{1}\left(m\left(S, R P^{2} ; f_{2 k}\right)\right)=\pi_{1}\left(\Gamma\left(S, E P \xi ; f_{2 k}\right)\right.$. The Euler class is $\chi\left(\eta^{\prime}\right)=\chi(\eta)=d\left(f_{2 k}\right)=2 k$, while the first SW class is $w_{1}\left(\eta^{\prime}\right)=w_{1}(\eta)=$ $f_{2 k}^{*}\left(w_{1}\left(\tau \mathrm{R} P^{2}\right)\right)=\theta_{0}\left(w_{1}\left(\tau \mathrm{R} P^{2}\right)\right)=w_{1}(\tau S)=0$

We need to distinguish between the two cases $\chi(\eta)=0$ or not, i.e. $k=0$ or $k \neq 0$.

Case 1.1. $k \neq 0$. By (2) of Theorem 2.5, since $\chi\left(\eta^{\prime}\right) \neq 0$, we have that

$$
\pi_{1}\left(\Gamma\left(S, P \xi ; f_{2 k}\right)\right)=\pi_{1}\left(\Gamma\left(S, S \xi^{\prime} ; f_{2 k}^{\prime}\right)\right),
$$

so we use Theorem 2.4 in order to compute $\pi_{1}(\Gamma)=\pi_{1}\left(\Gamma^{\prime}\right)=\pi_{1}\left(\Gamma\left(S, S \xi^{\prime}\right.\right.$; $\left.f_{2 k}^{\prime}\right)$ ). We have the exact sequence

$$
\begin{gathered}
H^{0}\left(S ; \mathbf{Z}^{T}\left[w_{1}(\eta)\right]\right) \longrightarrow H^{2}(S ; \mathbf{Z}) \stackrel{\phi}{\longrightarrow} \pi_{1}\left(\Gamma^{\prime}\right) \stackrel{p}{\longrightarrow} H^{1}\left(S ; \mathbf{Z}^{T}\left[w_{1}(\eta)\right]\right) \longrightarrow 0, \\
\mathrm{Z} \stackrel{2 k}{\longrightarrow} \mathrm{Z}[\Lambda] \stackrel{\phi}{\longrightarrow} \pi_{1}\left(\Gamma^{\prime}\right) \stackrel{p}{\longrightarrow} \mathrm{Z}\left[\bar{a}_{1}, \bar{b}_{1}, \ldots, \bar{a}_{g}, \bar{b}_{g}\right] \longrightarrow 0 .
\end{gathered}
$$

In order to find the extension, we take a set of generators

$$
\left\{\alpha_{1}, \beta_{1}, \ldots, \alpha_{g}, \beta_{g}, \lambda\right\},
$$


such that

$$
\begin{aligned}
p\left(\alpha_{i}\right) & =\bar{a}_{i}, \\
p\left(\beta_{i}\right) & =\bar{b}_{i}, \\
\lambda & =\phi(\Lambda) .
\end{aligned}
$$

Now, by (1) of Theorem $2.4, \lambda$ is central. Since $2 k \Lambda$ is in the image of the previous homomorphism, it must be in the kernel of $\phi$, namely $\phi(2 k \Lambda)=1$; as $\phi(2 k \Lambda)=\lambda^{2 k}$, it follows $\lambda^{2 k}=1$. By (2) of the same theorem,

$$
\begin{aligned}
{\left[\alpha_{i}, \alpha_{j}\right] } & =\phi\left(2 \bar{a}_{i} \cup \bar{a}_{j}\right)=\phi(0)=1, \\
{\left[\beta_{i}, \beta_{j}\right] } & =\phi\left(2 \bar{b}_{i} \cup \bar{b}_{j}\right)=\phi(0)=1, \\
{\left[\alpha_{i}, \beta_{j \neq i}\right] } & =\phi\left(2 \bar{a}_{i} \cup \bar{b}_{j \neq i}\right)=\phi(0)=1, \\
{\left[\alpha_{i}, \beta_{i}\right] } & =\phi\left(2 \bar{a}_{i} \cup \bar{b}_{i}\right)=\phi(2 \Lambda)=\lambda^{2} .
\end{aligned}
$$

Therefore

$$
\begin{gathered}
\pi_{1}(\Gamma)=\left\langle\alpha_{1}, \beta_{1}, \ldots, \alpha_{g}, \beta_{g}, \lambda ;\left[\alpha_{j}, \beta_{j}\right]=\lambda^{2}, \lambda^{2 k}=1\right. \\
\left.\left[\alpha_{j}, \alpha_{k}\right]=\left[\beta_{j}, \beta_{k}\right]=\left[\alpha_{j}, \beta_{k \neq j}\right]=\left[\alpha_{j}, \lambda\right]=\left[\beta_{j}, \lambda\right]=1\right\rangle .
\end{gathered}
$$

Note the extension is the abelian extension when $k=1$.

Case 1.2. $k=0$. Note that this is the case of the constant map $f_{0}=c_{0}$. By (2) of Theorem 2.5, since $\chi\left(\eta^{\prime}\right)=0$, we have that

$$
\pi_{1}(\Gamma)=\pi_{1}\left(\Gamma\left(S, P \xi ; f_{0}\right)\right) \neq \pi_{1}\left(\Gamma^{\prime}\right)=\pi_{1}\left(\Gamma\left(S, S \xi^{\prime} ;\left(f_{0}\right)^{\prime}\right)\right),
$$

but can be determined using the extension given in the same theorem.

First, we use Theorem 2.4 in order to compute $\pi_{1}\left(\Gamma^{\prime}\right)$. We are exactly in the same situation as above in Case 1.1, thus we obtain

$$
\begin{aligned}
\pi_{1}\left(\Gamma^{\prime}\right)=\left\langle\alpha_{1}, \beta_{1}, \ldots, \alpha_{g}, \beta_{g}, \lambda ;\left[\alpha_{j}, \beta_{j}\right]=\lambda^{2}, \lambda^{2 k}=1,\right. \\
{\left.\left[\alpha_{j}, \alpha_{k}\right]=\left[\beta_{j}, \beta_{k}\right]=\left[\alpha_{j}, \beta_{k \neq j}\right]=\left[\alpha_{j}, \lambda\right]=\left[\beta_{j}, \lambda\right]=1\right\rangle . }
\end{aligned}
$$


Second, we use the exact sequence given in Theorem 2.5.

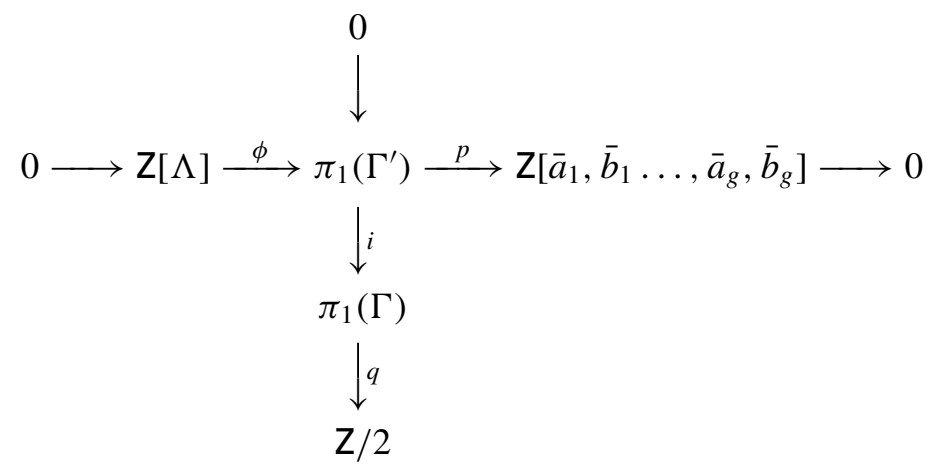

Consider the set of generators

$$
\left\{\hat{\alpha}_{1}, \hat{\beta}_{1}, \ldots, \hat{\alpha}_{g}, \hat{\beta}_{g}, \hat{\lambda}, t\right\}
$$

such that

$$
\begin{aligned}
\hat{\alpha}_{j} & =i\left(\alpha_{j}\right), \\
\hat{\beta}_{i} & =i\left(\beta_{i}\right), \\
\hat{\lambda} & =i(\lambda), \\
q(t) & =1 .
\end{aligned}
$$

We have the following facts:

- since $w_{1}(\eta)=0$, by (3d) of Theorem $2.5, t^{2}=1$;

- all the relations of $\pi_{1}\left(\Gamma^{\prime}\right)$ survive since $i$ is homomorphism;

- by (3a) of Theorem $2.5, \hat{\lambda}$ is central, and therefore $[t, \hat{\lambda}]=1$;

- by (3c) of Theorem 2.5 , since the square cup product of each two elements is 0 , we have

$$
t \hat{\alpha}_{i} t \hat{\alpha}_{i}=t \hat{\beta}_{i} t \hat{\beta}_{i}=1 \text {. }
$$

Thus, we have proved that

$$
\begin{gathered}
\pi_{1}(\Gamma)=\left\langle\alpha_{1}, \beta_{1}, \ldots, \alpha_{g}, \beta_{g}, \lambda, t\right. \\
{\left[\alpha_{j}, \alpha_{k}\right]=\left[\beta_{j}, \beta_{k}\right]=\left[\alpha_{j}, \beta_{k \neq j}\right]=\left[\alpha_{j}, \lambda\right]=\left[\beta_{j}, \lambda\right]=[t, \lambda]=1,} \\
\left.t^{2}=1,\left[\alpha_{j}, \beta_{j}\right]=\lambda^{2}, t \alpha_{j} t \alpha_{j}=t \beta_{j} t \beta_{j}=1\right\rangle .
\end{gathered}
$$

Part 2. $\pi_{1}\left(m\left(S, \mathrm{R} P^{2} ; f_{ \pm x}\right)\right)=\pi_{1}\left(\Gamma\left(S, E P \xi ; f_{ \pm x}\right)\right.$. The Euler class is $\chi\left(\eta^{\prime}\right)=\chi(\eta)=d\left(f_{ \pm x}\right)=0$, while the first SW class is $w_{1}\left(\eta^{\prime}\right)=w_{1}(\eta)=$ 
$f_{ \pm x}^{*}\left(w_{1}\left(\tau \mathrm{R} P^{2}\right)\right)=\theta\left(w_{1}\left(\tau \mathrm{R} P^{2}\right)\right)=x \neq 0$, since $\theta \neq \theta_{0}=0$. By (2) of Theorem 2.5, since $\chi\left(\eta^{\prime}\right)=0$, we have that

$$
\pi_{1}(\Gamma)=\pi_{1}\left(\Gamma\left(S, P \xi ; f_{ \pm x}\right)\right) \neq \pi_{1}\left(\Gamma^{\prime}\right)=\pi_{1}\left(\Gamma\left(S, S \xi^{\prime} ;\left(f_{ \pm x}\right)^{\prime}\right)\right) .
$$

First, we use Theorem 2.4 in order to compute $\pi_{1}\left(\Gamma^{\prime}\right)$. We have the exact sequence

$$
\begin{gathered}
H^{0}\left(S ; \mathbf{Z}^{T}[x]\right) \longrightarrow H^{2}(S ; \mathbf{Z}) \stackrel{\phi}{\longrightarrow} \pi_{1}\left(\Gamma^{\prime}\right) \stackrel{p}{\longrightarrow} H^{1}\left(S ; \mathbf{Z}^{T}[x]\right) \longrightarrow 0 \\
0 \longrightarrow \mathrm{Z}[\Lambda] \stackrel{\phi}{\longrightarrow} \pi_{1}\left(\Gamma^{\prime}\right) \stackrel{p}{\longrightarrow} \mathrm{Z}\left[\bar{a}_{1}, \bar{b}_{1}, \ldots, \bar{a}_{g-1}, \bar{b}_{g-1}\right] \oplus \mathbf{Z} / 2[\bar{x}] \longrightarrow 0 .
\end{gathered}
$$

In order to find the extension, we take a set of generators

$$
\left\{\alpha_{1}, \beta_{1}, \ldots, \alpha_{g-1}, \beta_{g-1}, \lambda, \delta\right\},
$$

such that

$$
\begin{aligned}
p\left(\alpha_{i}\right) & =\bar{a}_{i}, \\
p\left(\beta_{i}\right) & =\bar{b}_{i}, \\
p(\delta) & =\bar{x}, \\
\lambda & =\phi(\Lambda) .
\end{aligned}
$$

We have the following facts.

- By (1) of Theorem 2.4, $\lambda=\phi(\Lambda)$ is central.

- By (3) of Theorem 2.4, there exists an element $y \in \pi_{1}\left(\Gamma^{\prime}\right)$, such that

$$
p(y)=\bar{x}, \quad y^{2}=\phi(\bar{x} \cup \bar{x}+z),
$$

where $z \in H^{2}(S ; \mathbf{Z})=\mathbf{Z}[\Lambda]$, with $z \bmod 2=w_{2}(\eta)$. Since, $w_{2}(\eta)=$ $\chi(\eta) \bmod 2=0 \bmod 2=0$, we have $z=2 n \Lambda$. Since $\bar{x} \cup \bar{x}$ is in $Z$ and $\bar{x}$ has order 2, it follows that $\bar{x} \cup \bar{x}=0$. Therefore, $y^{2}=\phi(2 n \Lambda)=\lambda^{2 n}$. But $p(y)=p(\delta)$, and therefore $y=\delta \lambda^{k}$, for some $k$. This means that $y^{2}=\delta^{2} \lambda^{2 k}$ (since $\lambda$ is central), and therefore $\lambda^{2 n}=\delta^{2} \lambda^{2 k}$. Thus, we have got the relation $\delta^{2}=\lambda^{2 m}$, and any element of the form $\delta \lambda^{k}$ can be chosen as $y$. Since the group is isomorphic to the one with the unique relation $\delta^{2}=1$, and with the same range of choice for $y$, we take the last simpler relation and we set $y=\delta$ (the explicit choice of $y$ is necessary in what follows).

- By (2) of Theorem 2.4,

$$
\begin{aligned}
& {\left[\alpha_{j}, \delta\right]=\phi\left(2 \bar{a}_{j} \cup \bar{x}\right)=\phi\left(\bar{a}_{j} \cup \overline{2} x\right)=\phi(0)=1,} \\
& {\left[\beta_{j}, \delta\right]=\phi\left(2 \bar{b}_{j} \cup \bar{x}\right)=\phi\left(\bar{b}_{j} \cup \overline{2} x\right)=\phi(0)=1,}
\end{aligned}
$$


since $\bar{x}$ has order 2 . Also

$$
\begin{aligned}
{\left[\alpha_{j}, \alpha_{k}\right] } & =\phi\left(2 \bar{a}_{j} \cup \bar{a}_{k}\right)=\phi(0)=1, \\
{\left[\beta_{j}, \beta_{k}\right] } & =\phi\left(2 \bar{b}_{j} \cup \bar{b}_{k}\right)=\phi(0)=1, \\
{\left[\alpha_{j}, \beta_{k \neq j}\right] } & =\phi\left(2 \bar{a}_{j} \cup \bar{b}_{k \neq j}\right)=\phi(0)=1, \\
{\left[\alpha_{j}, \beta_{j}\right] } & =\phi\left(2 \bar{a}_{j} \cup \bar{b}_{j}\right)=\phi(2 \Lambda)=\lambda^{2} .
\end{aligned}
$$

Therefore

$$
\begin{aligned}
& \pi_{1}\left(\Gamma^{\prime}\right)=\left\langle\alpha_{1}, \beta_{1}, \ldots, \alpha_{g-1}, \beta_{g-1}, \delta, \lambda ;\right. \\
& {\left[\alpha_{j}, \alpha_{k}\right]=\left[\beta_{j}, \beta_{k}\right]=\left[\alpha_{j}, \beta_{k \neq j}\right]=\left[\alpha_{j}, \lambda\right]=\left[\beta_{j}, \lambda\right]=[\delta, \lambda]=1,} \\
& \left.\left[\alpha_{j}, \beta_{j}\right]=\lambda^{2}, \delta^{2}=1\right\rangle .
\end{aligned}
$$

Note that we have proved that $\pi_{1}\left(\Gamma^{\prime}\right)$ is the direct sum of $Z / 2$ plus one extension $G$ of

$$
\mathrm{Z} \rightarrow G \rightarrow(2 g-2) \mathrm{Z}
$$

Second, we use the exact sequence given in Theorem 2.5.

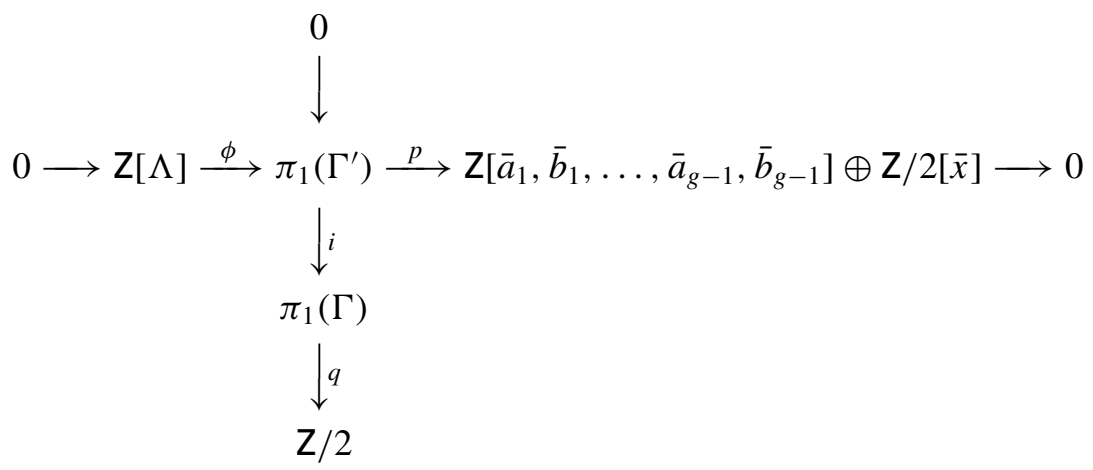

Consider the set of generators

$$
\left\{\hat{\alpha}_{1}, \ldots, \hat{\alpha}_{g-1}, \hat{\beta}_{1}, \ldots, \hat{\beta}_{g-1}, \hat{\delta}, \hat{\lambda}, t\right\}
$$

such that

$$
\begin{aligned}
\hat{\alpha}_{j} & =i\left(\alpha_{j}\right), \\
\hat{\beta}_{j} & =i\left(\beta_{j}\right), \\
\hat{\lambda} & =i(\lambda), \\
\hat{\delta} & =i(\delta) \\
q(t) & =1 .
\end{aligned}
$$


Since $i$ is homomorphism, all the relations of $\pi_{1}\left(\Gamma^{\prime}\right)$ survive. Since $w_{1}(\eta)=$ $x \neq 0$, by (3e) of Theorem $2.5, t^{2}=i(y)=\hat{\delta}$, and since $\hat{\delta}^{2}=1, t^{4}=1$. Also, by (3a) of the same theorem, $\hat{\lambda}$ is central, and this implies that $[\hat{\lambda}, t]=1$. By (3c) of Theorem 2.5, since all the cup square are trivial,

$$
t \hat{\alpha}_{j} t^{-1} \hat{\alpha}_{j}=t \hat{\beta}_{j} t \hat{\beta}_{j}=1 \text {. }
$$

Thus, we have proved that

$$
\begin{gathered}
\pi_{1}(\Gamma)=\left\langle\alpha_{1}, \beta_{1}, \ldots, \alpha_{g-1}, \beta_{g-1}, \lambda, t\right. \\
{\left[\alpha_{j}, \alpha_{k}\right]=\left[\beta_{j}, \beta_{k}\right]=\left[\alpha_{j}, \beta_{k \neq j}\right]=\left[\alpha_{j}, \lambda\right]=\left[\beta_{j}, \lambda\right]=[t, \lambda]=1,} \\
\left.t^{4}=1,\left[\alpha_{j}, \beta_{j}\right]=\lambda^{2}, t \alpha_{j} t^{-1} \alpha_{j}=t \beta_{j} t \beta_{j}=1\right\rangle .
\end{gathered}
$$

\section{The 2-sphere}

THEOREM 5.1.

$$
\pi_{1}\left(m\left(S^{2}, \mathrm{R} P^{2} ; f_{2 k}\right)\right)= \begin{cases}\mathbf{Z} / 2 k, & \text { if } k>0, \\ \mathbf{Z} \oplus \mathbf{Z} / 2, & \text { if } k=0 .\end{cases}
$$

Proof. By Proposition 2.1, we have

$$
m\left(S^{2}, \mathrm{R} P^{2}\right)=\bigsqcup_{k=0}^{\infty} m\left(S^{2}, \mathrm{R} P^{2} ; f_{2 k}\right),
$$

and

$$
\left(f_{2 k}\right)_{*}=\theta_{0}=0 .
$$

The Euler class is $\chi\left(\eta^{\prime}\right)=\chi(\eta)=d\left(f_{2 k}\right)=2 k$, while the first SW class is $w_{1}\left(\eta^{\prime}\right)=w_{1}(\eta)=f_{2 k}^{*}\left(w_{1}\left(\tau \mathrm{R} P^{2}\right)\right)=\theta_{0}\left(w_{1}\left(\tau \mathrm{R} P^{2}\right)\right)=w_{1}\left(\tau S^{2}\right)=0$. We distinguish between the two cases $\chi(\eta)=0$ or not.

Case $1 . k \neq 0$. By (2) of Theorem 2.5,

$$
\pi_{1}(\Gamma)=\pi_{1}\left(\Gamma\left(S, P \xi ; f_{2 k}\right)\right)=\pi_{1}\left(\Gamma\left(S, S \xi^{\prime} ; f_{2 k}^{\prime}\right)\right),
$$

and we have the exact sequence

$$
\mathrm{Z} \stackrel{2 k}{\longrightarrow} \mathrm{Z}[\Lambda] \stackrel{\phi}{\longrightarrow} \pi_{1}\left(\Gamma^{\prime}\right) \stackrel{p}{\longrightarrow} 0 \longrightarrow 0 .
$$

Thus, we have $\pi_{1}(\Gamma)=\mathrm{Z} / 2 k[\lambda]$, where $\lambda=\phi(\Lambda)$.

Case 2. $k=0$. This is the case of the constant map $f_{0}=c_{0}$. Now, $\chi\left(\eta^{\prime}\right)=0$, so (2) of Theorem 2.5 implies that

$$
\pi_{1}(\Gamma)=\pi_{1}\left(\Gamma\left(S, P \xi ; f_{0}\right)\right) \neq \pi_{1}\left(\Gamma^{\prime}\right)=\pi_{1}\left(\Gamma\left(S, S \xi^{\prime} ;\left(f_{0}\right)^{\prime}\right)\right) .
$$


By Theorem 2.4, we have the exact sequence

$$
\mathrm{Z} \stackrel{0}{\longrightarrow} \mathrm{Z}[\Lambda] \stackrel{\phi}{\longrightarrow} \pi_{1}^{\prime} \stackrel{p}{\longrightarrow} 0 \longrightarrow 0 .
$$

Thus, $\pi_{1}\left(\Gamma^{\prime}\right)=\mathrm{Z}[\lambda]$, with $\phi(\Lambda)=\lambda$. By Theorem 2.5, we have the exact sequence

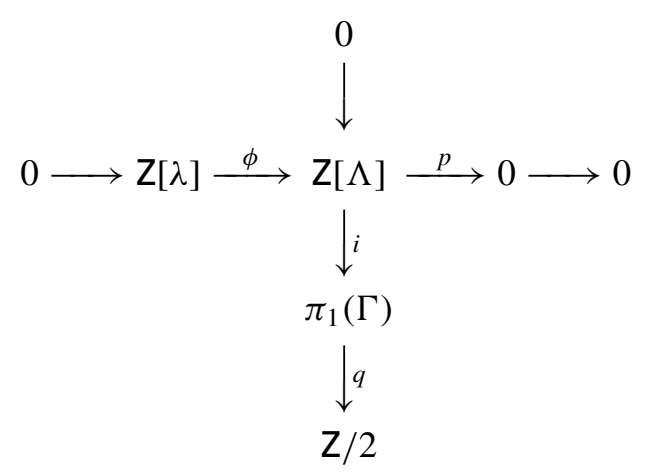

Consider the set of generators $\{\hat{\lambda}, t\}, \hat{\lambda}=i(\lambda)$, and $q(t)=1$. Since $w_{1}(\eta)=0$, by $(3 \mathrm{e})$ of Theorem $2.5, t^{2}=1$. Also, all the relations of $\pi_{1}\left(\Gamma^{\prime}\right)$ survive. Thus the extension is abelian. And because of the previous relation, it is

$$
\pi_{1}(\Gamma)=\mathbf{Z}[\lambda] \oplus \mathbf{Z} / 2[t]
$$

REMARK 5.2. It is worthwhile to mention that all the results above in the case where $\theta$ is the trivial homomorphism follows from a stronger result, based on a very simple observation. In fact, the natural map $m\left(S, S^{2} ; f_{k}\right) \rightarrow$ $m\left(S, \mathrm{R} P^{2} ; f_{|2 k|}\right)$, obtained by composing with the projection $S^{2} \rightarrow \mathrm{R} P^{2}$, is a homeomorphism for $S$ orientable and $\left(f_{|2 k|}\right)_{*}=\theta=\theta_{0}$. A similar statement is true for $S$ nonorientable. Namely the maps $m\left(S, S^{2} ; f_{0}\right) \rightarrow m\left(S, \mathrm{R} P^{2} ; f_{0}^{0}\right)$ and $m\left(S, S^{2} ; f_{-0}\right) \rightarrow m\left(S, \mathrm{R} P^{2} ; f_{-0}^{0}\right)$ obtained by composing with the projection $S^{2} \rightarrow \mathrm{R} P^{2}$, are double coverings for $S$ nonorientable, since again $\left(f_{ \pm 0}^{0}\right)_{*}=\theta_{0}$; here, $f_{0}$ is the constant map and $f_{-0}$ is a non null homotopic map from $S$ to $S^{2}$. Hence, all the results from [7] can be applied to describe the homotopy type of these components of the spaces $m\left(S, \mathrm{R} P^{2}\right)$. It is easy to see that this is compatible with our calculation.

ACKNOWLEDGments. We would like to thank the referee for his useful remarks and for suggesting some interesting related works. 


\section{REFERENCES}

1. Crabb, M., Spreafico, M., and Sutherland, W., Enumerating projectively equivalent bundles, Math. Proc. Cambridge Philos. Soc. 125 (1999), 223-242.

2. Epstein, D. B. A., The degree of a map, Proc. London Math. Soc. 16 (1966), 369-383.

3. Gottlieb, D. H., Covering transformation and universal fibrations, Illinois J. Math. 13 (1969), 432-437.

4. Hansen, V. L., On the space of maps of a closed surface into the 2-sphere, Math. Scand. 35 (1974), 149-158.

5. Hansen, V. L., Spaces of maps into Eilenberg-MacLane spaces, Canad. J. Math. XXXIII (1981), 782-785.

6. Hansen, V. L., The homotopy groups of a space of maps between oriented closed surfaces, Bull. London Math. Soc. 15 (1983), 360-364.

7. Hansen, V. L., The space of self-maps on the 2-sphere. Groups of self-equivalences and related topics, Lecture Notes in Math. 1425 (1990), 40-47.

8. Hansen, V. L., The homotopy problem for the components in the space of maps on the n-sphere, Quart. J. Math. 25 (1974), 313-321.

9. Larmore, L. L., and Thomas, E., On the fundamental group of spaces of sections, Math. Scand. 10 (1980), 232-246.

10. Møller, J. M., On spaces of maps between complex projective spaces, Proc. Amer. Math. Soc. 91 (1984), 471-476.

11. Olum, P., Mappings of manifolds and the notion of degree, Ann. of Math. 72 (1953), 458-480.

12. Sutherland, W. A., Path-components of function spaces, Quart. J. Math. 34 (1983), 223-233.

13. Yamanoshita, T., On the space of self-homotopy equivalences of the projective plane, J. Math. Soc. Japan 45 (1993), 489-494.

DEPARTAMENTO DE MATEMÁTICA-IME-USP CAIXA POSTAL 66281-AG. CIDADE DE SÃO PAULO CEP: 05315-970 - SÃO PAULO-SP BRASIL

E-mail: dlgoncal@ime.usp.br
DEPARTAMENTO DE MATEMÁTICA-ICMC UNIVERSITY OF SÃO PAULO-SÃO CARLOS CAIXA POSTAL 668, SÃO CARLOS-SP BRASIL

E-mail: mauros@icmc.usp.br 